\title{
Inverse Correlation of miR-27a-3p and CDH5 Expression Serves as a Diagnostic Biomarker of Proliferation and Metastasis of Clear Cell Renal Carcinoma
}

\author{
Yifang Wang \\ Guangxi Medical University \\ Xiaohui Zhou \\ Guangxi Medical University \\ Peipei Han \\ Guangxi Medical University \\ Yunliang Lu \\ guangxi medical university \\ Xuemin Zhong \\ Guangxi Medical University \\ Yanping Yang \\ Guangxi Medical University \\ Danping Li \\ Guangxi Medical University \\ Deling Liu \\ Guangxi Medical University \\ Qiuyun Li \\ Guangxi Medical University \\ Nenghui Pan \\ Guangxi Medical University \\ Yingxi Mo \\ Guangxi Medical University \\ Wenqi Luo \\ Affiliated Tumor Hospital of Guangxi Medical University
}

Ping Li

Guangxi Medical University

Xiaoying Zhou ( $\sim$ zhouxiaoying1982@foxmail.com )

Life Science Institute, Guangxi Medical University. 530021, \#22 Shuangyong Road, Nanning, China https://orcid.org/0000-0003-3298-5089

Liudmila Matskova

Immanuel Kant Baltic Federal University

Research article

Keywords: ccRCC, CDH5, mir-27a-3p, biomarker

Posted Date: September 10th, 2020

DOl: https://doi.org/10.21203/rs.3.rs-67712/v1

License: @ (1) This work is licensed under a Creative Commons Attribution 4.0 International License. Read Full License

Version of Record: A version of this preprint was published at Pathology - Research and Practice on April 1st, 2021. See the published version at https://doi.org/10.1016/j.prp.2021.153393. 


\section{Abstract}

Background: Cadherin-5 (CDH5) is aberrantly expressed in a variety of human cancers and plays an important role in angiogenesis. But its role in renal clear cell carcinoma (ccRCC) remains to be understood.

Methods: The dysregulation of CDH5 expression in CCRCC and its association with clinicopathological characteristics were analyzed using the TCGA database. A meta-analysis was performed to verify the alteration of CDH5 expression in CCRCC using the GEO database.

Quantitative RT-PCR and immunohistochemical staining were applied to assess the transcriptional and protein levels of CDH5. TargetScan and Tarbase were employed to predict the miRNAs with the potential to target mRNA of CDH5.

Results: The mRNA level of CDH5 in ccRCC was significantly higher than in normal tissue. CDH5 mRNA expression could therefore serve as a potential diagnostic biomarker for $\mathrm{CCRCC}(\mathrm{AUC}=0.844)$. However, the reduced $\mathrm{CDH} 5$ transcription levels were significantly correlated with patients in the T3-4 stage, lymph node and distant metastasis, as well as with a worse clinical outcome. We further observed that CDH5, at the protein level, was almost absent in ccRCC samples. In addition, a few databases screen showed that mir-27a-3p, is a highly conserved miRNA targeting CDH5. The expression of mir-27a-3p was significantly elevated in ccRCC tissues in contrast to normal tissues. Importantly, it was positively associated with the T3-4 stage and M stage, respectively, suggesting that the expression level of mir-27a-3p could serve as a diagnostic biomarker for ccRCC (AUC=0.775).

Conclusion: Our data suggest that the reduced translational levels of CDH5 in ccRCC was related to the overexpression of mir-27a-3p. The higher mir-27a-3p and lower CDH5 expression significantly correlated with advanced clinical stages for ccRCC patients.

\section{Background}

The current incidence of renal cell carcinoma (RCC) increases by $2 \%$ per year.[1] By the end of 2020, it is estimated that there will be 73,750 new cases of RCC and about 14,830 new deaths $[2,3]$. The common type of RCC is clear cell renal cell carcinoma (ccRCC), and the mortality rate of ccRCC was about 4.1 per 100,000.[4] Partial and radical nephrectomy is the main method of therapy for localized ccRCC. However, even after surgery , $30 \%$ of the patients have distant metastases within five years, which is the main cause of treatment failure.[5] ccRCC had been shown largely refractory to conventional cytotoxic chemo and hormone-therapy, while the effect of radiotherapy is a limited due to a low response rate.[6] It is necessary to identify new molecular biomarkers for early diagnosis and precise treatment.

Cadherins are cell adhesion molecules that are responsible for the normal structure of epithelial tissues. They also play a vital role in cell differentiation and tissue remodeling.[7] The alteration of cadherin expression leads to tumor initiation and progression.[8] The aberrant expression of E-cadherin ( $\mathrm{CDH} 1)$ and $\mathrm{N}$-cadherin $(\mathrm{CDH} 2)$ is closely related to the epithelial-to-mesenchymal transition (EMT) in various tumors, such as lung, colorectal and prostate cancer and others.[9-11] Expression of these cadherins also serves as reliable prognostic biomarkers.

$\mathrm{CDH} 5$, also known as VE-cadherin, was mainly expressed in the inter-endothelia junction. The CDH5 protein participates in maintaining the integrity of endothelial and vascular tissues, functioning as a regulator in signaling of endothelial cell during vessel morphogenesis and vascular development.[12] Therefore, the dysregulation of CDH5 expression promotes tumor progression by stimulating angiogenesis.[13] In breast cancer, the levels of $\mathrm{CDH} 5$ protein expression can be used to distinguish breast cancer patients from non-breast cancer patients and its high expression is predictive of metastasis in breast cancer.[14] In gastric cancer, the up-regulation of CDH5 protein expression is associated with poor outcomes for patients.[15] The altered expression of $\mathrm{CDH} 5$ protein in glioblastoma multiforme is suggested to contribute to the tumorigenic process by facilitating new blood vessel formation.[16] In highly aggressive melanoma cells, CDH5 protein acts as a co-regulatory element of an EphA2 complex to control tumor neovascularization.[17] However, the clinical significance of CDH5 mRNA expression levels has not been studied yet.

MiRNAs, are highly conserved non-coding single-stranded RNAs of 22 nucleotides length, which recognize the 3' untranslated region (3'-UTR) of target mRNAs and either prevent their translation or facilitate their degradation, depending on partial or complete complementarity to the targeted nucleotide sequence. In other words, miRNAs regulate gene expression at the post-transcription level. [18] Several members of the $\mathrm{CDH}$ family genes has already been shown to be regulated by miRNAs and this mechanism was shown to be involved in cancer progression and metastasis. For example, $\mathrm{CDH} 1$ is a direct target for MiR-217, it was shown that miR-217 overexpression can enhance gastric cancer cell proliferation and reduce exosome $\mathrm{CDH} 1$ levels.[19] In non-small cell lung cancer, miR-124 can inhibit CDH2 expression and regulate the EMT. [20] The clinical significance of CDH5 mRNA expression and its control by a miRNA expression in ccRCC has not been shown yet.

In this study, we aim to analyze the expression of $\mathrm{CDH} 5$ at the gene and the protein level and its regulation by expression of miRNA-27a-3p in ccRCC. We also evaluated their potential significance role as diagnostic and prognostic biomarkers. In addition, the expressional levels of the CDH5 gene and miRNA-27a-3p were analyzed to correlate with the clinical characteristics of patients with ccRCC.

Page $2 / 17$ 


\section{Materials And Methods}

\section{Analysis of the TCGA database on the transcriptional level of CDH5}

The TCGA database was downloaded from the UCSC Cancer Genomics Browser (https://xena.ucsc.edu/). The RNA sequencing data for 533 cCRCC patients and 72 noncancerous tissues together with complete clinical information on age, gender, pathologic stage and others for all subjects from the TCGA database were analyzed. Subsequently, the correlation between the mRNA level of CDH5 and clinical characteristics of ccRCC patients was analyzed.

\section{A meta-analysis based on the GEO datasets}

The alteration of CDH5 mRNA level in cCRCC was further investigated using the GEO database, with search terms as follows: 1) "renal cancer", 2) "kidney OR renal AND cancer OR carcinoma OR tumor OR neoplasm* OR malignant* OR ccRCC OR RCC OR RCCC". The microarrays data was used to compare the $\mathrm{CDH} 5 \mathrm{mRNA}$ level in ccRCC and normal tissues, by meta-analysis. The criteria of the microarrays data inclusion into the analysis were: 1) more than three tumor samples and three normal controls on the microarray, 2) the human tissues served as a source for microarrays. The STATA 12 software was used for meta-analysis.

\section{Cell lines and tissue samples}

The human epithelial cell lines established human cells 293T (CRL-11268, ATCC, USA) and tumorous kidney-ACHN (ZQ0340, CHINA) were cultured in Dulbecco's modified Eagle's medium (DMEM; Gibco, USA) supplemented with 10\% fetal bovine serum (FBS; Invitrogen) and penicillin $(100 \mathrm{U} / \mathrm{ml})$. The cell lines established from ccRCC patients- 786-0 (CRL-1932, ATCC, USA) and OSRC-2 were maintained in 1640 medium (Invitrogen, Carlsbad, CA, USA) supplemented with 10\% fetal bovine serum (FBS; Invitrogen) and penicillin (100 U/ml). The Caki-2 (HTB-47, ATCC, USA) was cultured in McCoy's 5a Medium Modified supplemented with 10\% fetal bovine serum (FBS; Invitrogen) and penicillin $(100 \mathrm{U} / \mathrm{ml})$. All cell lines were maintained at $5 \% \mathrm{CO}_{2}$, at $37 \circ \mathrm{C}$.

The cDNA microarray containing samples of ccRCC $(n=15)$ and matched adjacent tissues were purchased from Shanghai OUTDO Biotech Co., Ltd. (Shanghai, China; Cat no: me cDNA-HKidE030CS01). The array was supplemented with corresponding clinicopathological information, including gender, age, pathological classification, and distant metastasis.

Seventeen cases of clear cell carcinoma of kidney and adjacent tissues were acquired from the Department of Pathology, Affiliated Tumor Hospital of Guangxi Medical University from January 2015 to March 2017. All patients signed informed consent forms.

\section{Real-time Quantitative RT-PCR}

The expression level of CDH5 mRNA in samples was detected in a Q-RT PCR reaction with the use of an SYBR Green quantitative PCR reagent kit (\#A25742, Thermo Fisher Scientific, Waltham, MA, USA), on a QuantStudio 6 Flex Real-Time PCR System (Thermo Fisher Scientific, Waltham, MA, USA). The expression levels were calculated by the 2- $\triangle \Delta C$ t method, in regards to the level of $\beta$-actin mRNA expression as the reference. The specific primers were as follows: CDH5-Forward, 5'-GGCTCCACAGAGCTCCACTC-3'; CDH5-Reverse, 5'TGAGGGATGTTTCTGTTCCGT-3'; $\beta$-actin-Forward, 5'-CTTCGCGGGCGACGAT-3'; $\beta$-actin-Reverse, 5'-CCACATAGGAATCCTTCTGACC-3'. The PCR reaction conditions were as follows: $95^{\circ} \mathrm{C}$ for $10 \mathrm{~min}$, followed by 40 cycles of $95^{\circ} \mathrm{C}$ for $15 \mathrm{~s}$, and $60^{\circ} \mathrm{C}$ for $60 \mathrm{~s}$.

\section{Immunohistochemistry (IHC) staining assay}

An SP-9000 detection kit (ZSGB-Bio, Beijing, China) was used for immunohistochemical staining on paraffin-embedded tissue slices. Tissues were deparaffinized and rehydrated in fresh xylene and gradient concentrations of alcohol, followed by antigenic thermal repair, followed by incubation with endogenous peroxidase blockers for 10 min. Primary antibodies against CDH5 (MABT129, Sigma, USA, 1:350 dilution) were added to the samples and incubated at $37^{\circ}$ for $1.5 \mathrm{~h}$. Next, samples were incubated for 10 min with secondary antibody coupled to horseradish peroxidase-labeled streptomycin for $10 \mathrm{~min}$, followed by incubation with the chromogenic horseradish peroxidase substrate 3,3'diaminobenzidine (DAB) reagent (ZLI-9018, ZSGB-BIO, Beijing, China). Hematoxylin was used for counterstaining. Images were acquired with an Olympus C-5050 microscope (Olympus, Japan).

All sections were evaluated by two pathologists independently on the clinicopathological characteristics of the samples. The ambiguous cases were discussed and classified based on consensus. Each slice was randomly divided into 5 areas and evaluated in multiples fields at $20 \times 10$ magnification. The scoring criteria were as previously described.[21]

\section{Prediction of miRNAs potentially targeting the CDH5 mRNA}


Potential miRNAs targeting the CDH5 mRNA were predicted using three publicly available programs: TargetScan (http://www.targetscan.org), Tarbase (http://carolina.imis.athena-innovation.gr) and miRBase (http://www.mirbase.org/).[22-24]

\section{Statistical analysis}

Statistical analysis was performed using the Statistical Program for Social Sciences (SPSS) 22.0 software. GraphPad Prism 6 software was employed for data imaging. The students' t-test was used to examine the correlation between CDH5 mRNA and miRNA expression data and clinicopathological parameters extracted from TCGA data on cCRCC. The significance of a difference between CDH5 mRNA expression values and the protein expression in cancer and normal tissues was evaluated with the use of the paired t-test. Diagnostic value for CDH5 mRNA expression in CCRCC was obtained after the analysis of receiver operating characteristic (ROC). A cut-off point was defined as the maximum Youden index. A P-value $<0.05$ was considered to be statistically significant.

\section{Results}

\section{The expression of CDH5 mRNA is altered in ccRCC.}

We compared the mRNA expression values for CDH5 in 533 samples of ccRCC and 72 normal samples in the TCGA database. We detected that CDH5 mRNA expression was elevated significantly in ccRCC as compared to normal tissues $(p<0.001$; Figure 1a).

To further explore the consistency of data, regarding the aberrant expression of CDH5 mRNA, we performed qRT-PCR analysis for CDH5 expression with cell lines established from kidney and cCRCC tissues as a source. We showed that, in contrast to human cells $293 \mathrm{~T}$, the expression level of CDH5 mRNA in renal carcinoma cells was significantly higher ( $p<0.05$, Figure $1 \mathrm{~b})$. We obtained consistent results comparing the 15 paired cCRCC and adjacent normal tissues in qRT-PCR analysis $(p=0.0019$, Figure 1c).

This was confirmed further by a meta-analysis of cDNA microarrays using the GEO database. Totally 30 microarray studies were included for the meta-analysis, which in total contained 1,141 ccRCC and 651 normal samples. In 28 out of 30 microarrays, a higher value was observed in ccRCC tissues as compared to normal tissues. To obtain the result we used a random-effects model, which provides the pooled Standard Mean Difference (SMD) value as 1.07, corresponding to $95 \%$ of confidence interval ( $\mathrm{Cl}, 0.96-1.19$, Figure $2 \mathrm{a})$, and less than $0.001(\mathrm{I} 2=86.8 \%$ ) the $p$-value, after the heterogeneity test. The sensitivity analysis indicated that no single study led to significant bias in overall merger results (Figure 2b). In addition, no publication bias was found in the study (Begg's test: $p=0.116$, Figure $2 \mathrm{c}$ ). The relevant information related to these 30 microarrays is provided in Table 1.

Surprisingly, immunohistochemistry (IHC) analysis for CDH5 protein expression of ccRCC samples demonstrated the opposite results to CDH5 mRNA expression level in ccRCC. As shown in Figure 3a, we did not observe CDH5 in the cell membrane or cytoplasm of ccRCC, On the contrary, $\mathrm{CDH} 5$ was mainly distributed on the proximal convoluted tubules of adjacent normal kidney tissue samples, even in the distal convoluted tubules of normal kidney samples while in ccRCC samples CDH5 protein expression was not been identified. Furthermore, the positive expression rate for $\mathrm{CDH} 5$ in non-tumorous renal tissues was much higher than that in the cCRCC tissues, and CDH5 staining was strongly positive in almost all non-tumor specimens (Figure 3b).

\section{Low CDH5 mRNA expression corresponds to a tumor stage/metastasis stage of ccRCC.}

We analyzed whether the CDH5 mRNA expression changes with ccRCC development (Table 2). To our surprise, we found that the transcribed CDH5 was closely correlated with patients in T3-4 stages $(p=0.016), \mathrm{N}$ stage $(p=0.008)$, and M stage $(p=0.001)$, respectively. This means that $\mathrm{CDH} 5$ expression tends to decrease with the increase of tumor size and metastasis, however, CDH5 expression does not fall lower than that in normal tissues.

Although the expression of CDH5 protein normal tissue can be clearly observed in our IHC results, the CDH5 protein expression levels did not significantly correlate with the clinical parameters (Table 3). We infer, that this result might be due to our small sample size.

\section{The expression level of CDH5 mRNA can be used as a molecular marker for the diagnosis and prognosis of ccRCC development.}

A ROC curve built based on the analysis of CDH5 mRNA expression in 533 cases of ccRCC and 72 cases of normal kidney tissue (Figure $4 a$ ). This allowed to estimate the area under the curve (AUC) value as equal to $0.844((p<0.001)$, which suggests that CDH5 mRNA expression could serve as a molecular marker to distinguish the tumor tissues from the non-tumor ones, (the sensitivity and specificity as 0.73 and 0.931 , respectively). In addition, we have evaluated the overall survival (OS) and relapse-free survival (RFS) time using the Kaplan-Meier plotter, which revealed that low CDH5 mRNA expression was significantly associated with the shorter OS (Figure 4b, p=0.013). While, no statistically significant difference was observed with regards to RFS time in ccRCC (Figure4c, $p=0.133$ ). 
In order to investigate the discrepancies between low CDH5 protein and high CDH5 mRNA expression in ccRCC, we searched for putative miRNAs targeting $\mathrm{CDH} 5$ at the post-transcriptional level using TargetScan, Tarbase, and miRbase data. We found that CDH5 mRNA contains a seed site for miR-27a-3p (Figure 5a), which suggests that miR-27a-3p may be involved in the regulation of CDH5 mRNA expression. Previous studies have shown that mir-27a can promote cell proliferation and metastasis in ccRCC.[25] We downloaded the data regarding miR-27a-3p expression in TCGA and compared 240 ccRCC with 70 normal cases. The miR-27a-3p was overexpressed in ccRCC tumors compared to normal (Figure $5 b, p<0.001$ ). Although miR-27a-3p may not directly affect the transcription level of CDH5, it may be significantly related to the down-regulation of $\mathrm{CDH} 5$ protein level in ccRCC. We analyzed the relationship between miR-27a-3p miRNA and clinical information. We found that there was a similar to CDH5 mRNA expression alterations trend in ccRCC tissues $(9.54 \pm 0.53)$ and in the normal kidney tissues (8.96 \pm 0.63$)$, with statistical significance $(p<0.001)$. Interestingly, in contrast to the CDH5 mRNA expression level, the miR-27a-3p miRNA expression level was positively correlated with stage T3-4 $(p=0.026)$, stage $M(p<0.001)$, and clinicopathological stage $(p<0.007)$ in patients with ccRCC (Table 4). The AUC value, based on the ROC curve analysis 0.775 ( $p<0.001$ ), for the CDH5 miRNA also allowed to distinguish tumor from non-tumor tissue (Figure 6a). Therefore, we inferred that miR-27a-3p and CDH5 play an important role, perhaps jointly, in the occurrence and development of ccRCC. The analysis of miR-27a-3p expression, using the Kaplan-Meier plotter, did not allow to distinguish between ccRCC and normal tissue samples in regards to either OS or RFS times (Figure 6b, 6c).

\section{Discussion}

Previously it was shown that high $\mathrm{CDH} 5$ expression is associated mainly with angiogenesis during cancer.[26-28] However, we found a more complicated relationship between $\mathrm{CDH} 5 \mathrm{mRNA}$ expression and cancer progression. The expression of CDH5 mRNA is increased only at the early stage of ccRCC. During the progress of $c c R C C$, we detected a gradual loss of CDH5 mRNA expression. We infer that high CDH5 mRNA expression may be associated with the initiation of CCRCC, but further tumor proliferation and metastasis is associated with a gradual loss of $\mathrm{CDH} 5$ expression. We suggest that the loss of $\mathrm{CDH} 5$ mRNA expression may be due to increased miR-27a-3p expression. Post-transcriptional control of CDH5 expression is under intensive investigation currently.[29]

Other miRNAs reported being functional during ccRCC development, are miR-106a-5p, which was shown to bind and destroy PAK5 mRNA in cCRCC, resulting in a decrease of expression of an oncogene PAK5 protein.[30] Mir-148a targets AKT2 to inhibit cell growth, colony formation, and tumor growth of ccRCC.[31] We found that rise in miR-27a-3p expression is associated with the higher T stage and metastasis in ccRCC, which coincides with the low expression of CDH5 mRNA in ccRCC with the same clinicopathological features. We suggest that miR-27a-3p affects the occurrence and development of the disease by targeting CDH5 mRNA. It has been shown that miR-27a-3p is an important regulatory factor in a variety of cancers. It plays a carcinogenic role in renal cell carcinoma, cervical cancer, gastric adenocarcinoma, and breast cancer.[25, 32-34] Mir-27a-3p has been identified to promote tumor invasion and metastasis in gastric, colon, pancreatic, and nasopharyngeal cancers.[35-38] On the other hand, a tumor suppressor role has also been shown for miR-27a-3p in some cancers. For instance, it inhibits the proliferation of non-small cell lung cancer cells by targeting HOXB8.[39] In hepatocellular carcinoma (HCC), the miR27a-3p inhibits tumor metastasis by binding to 3'UTR of CDH5 mRNA, thus, inhibiting EMT. [40]. We also observed a negative correlation between CDH5 mRNA and miR-27a-3p expression in ccRCC. But we infer the role of miR-27a-3p as a proto-oncogene in ccRCC. The role of CDH5 in ccRCC pathogenesis needs further investigation.

$\mathrm{CDH} 5$ plays a central role in contact inhibition by transmitting information on cell density to the nucleus. Thus, it is the main guardian of vascular integrity.[41] Previous studies show that the expression of CDH5 has a profound influence on the invasion and metastasis in malignant tumors. $\mathrm{CDH} 5$ can promote tumor angiogenesis and tumor cell proliferation.[42] CDH5 mRNA is overexpressed at the early stages of cancer, which is consistent with our study.[43] Subsequently, the CDH5 mediated process of tumor cells infiltration into adjacent tissues facilitates penetration of the vascular endothelial barrier, and systemic dissemination tumor cells through blood vessels and lymphatics, thus contributing to acquisition of an EMT phenotype.[44] CDH5 can also affects the EMT process indirectly.[45] These studies support our findings and warrant further investigation of the interrelation between CDH5 mRNA and miR-27a-3p in ccRCC pathogenesis.

In the current study, we also found an inverse relationship between CDH5 and miR-27a-3p expression in ccRCC clinical samples. In addition, our exploration of the TCGA database, TargetScan, TarBase and miRbase data, provide compelling evidence supporting the relation between miR-27a-3p and CDH5. In the past, most of the studies on tumor markers were devoted to the study of single mRNAs or miRNAs alone. Our research aims to study the effect of interaction between mRNA and miRNA, to investigate possible new approaches to find clinically applicable tumor biomarkers of ccRCC.

\section{Conclusions}


Decreased CDH5 mRNA and increased miR-27a-3p expression was found to correlate significantly with the advanced stages of tumor development in ccRCC patients. The mir-27a-3p and CDH5 mRNA expression can be used as biomarkers and targets during diagnosis and treatment for $c c R C C$ patients. The mir-27a-3p/CDH5 mRNA axis in the pathogenesis of $c c R C C$ requires further investigation.

\section{Abbreviations}

CDH5: Cadherin-5

RCC: renal cell carcinoma

ccRCC: renal clear cell carcinoma

FBS: fetal bovine serum

qRT-PCR: Quantitative real-time PCR

DAB: Diaminobenzidine

SPSS: Statistical Program for Social Sciences

EMT: epithelial-to-mesenchymal transition

IHC: immunohistochemistry

\section{Declarations}

\section{Availability of data and materials}

The datasets used and/or analysed during the current study are available from the corresponding author on reasonable request.

\section{Acknowledgment}

We thank Dr. Gösta Winberg from Ludwig Institute for Cancer Research, Stockholm, for his useful comments.

\section{Funding}

This study was support by by the National Scientific Foundation of Guangxi Province (2020GXNSFAA159106). The funders had no role in study design, data collection and analysis, decision to publish, or preparation of the manuscript.

\section{Author information}

contributions:

Yifang Wang and Xiaohui Zhou performed most of the bioinformatic analysis, QPCR and IHC staining, prepared the draft of of manuscript; Deling Liu, Qiuyun Li, Nenghui Pan cultured the cells and collected samples; Peipei Han and Yunliang Lu carried out the meta-analysis; Xuemin Zhong, Yanping Yang performed the ROC and survival analysis; Danping Li, Wenqi Luo investigated the miRNA targeting CDH5; Yingxi Mo and Ping Li were responsible for scoring the IHC staining data; Xiaoying Zhou and Liudmila, Matskova conceived the study and revised the manuscript. All authors commented on previous versions of the manuscript and approved the final manuscript.

\section{Corresponding author}

Correspondence to Xiaoying Zhou and Liudmila Matskova.

\section{Ethics declarations}

\section{Ethics approval and consent to participate}

This study was approved by the Ethics Committee of the Affiliated Tumor Hospital of Guangxi Medical University (LW2020043).

\section{Consent for publication}

Not applicable. 
The authors declare no potential conflicts of interest.

\section{Additional information}

\section{Publisher's Note}

Springer Nature remains neutral with regard to jurisdictional claims in published maps and institutional affiliations.

\section{References}

1. Motzer RJ, Agarwal N, Beard C, Bhayani S, Bolger GB, Carducci MA et al. Kidney Cancer. 2011;9(9):960-77.

2. Siegel RL, Miller KD, Jemal A. Cancer statistics, 2020. CA Cancer J Clin. 2020;70(1):7-30. doi:10.3322/caac.21590.

3. Capitanio U, Bensalah K, Bex A, Boorjian SA, Bray F, Coleman J et al. Epidemiology of Renal Cell Carcinoma. Eur Urol. 2019;75(1):74-84. doi:10.1016/j.eururo.2018.08.036.

4. Capitanio U, Montorsi F. Renal cancer. Lancet. 2016;387(10021):894-906. doi:10.1016/S0140-6736(15)00046-X.

5. Mikami S, Oya M, Mizuno R, Kosaka T, Katsube KI, Okada YJMMM. Invasion and metastasis of renal cell carcinoma. 2014;47(2):63-7.

6. Drucker BJJCTR. Renal cell carcinoma: Current status and future prospects. 2005;31(7):0-545.

7. Ko KS, Arora PD, Bhide V, Chen A, McCulloch CA. Cell-cell adhesion in human fibroblasts requires calcium signaling. J Cell Sci. 2001;114(Pt 6):1155-67.

8. Cavallaro U, Christofori GJANYAS. Multitasking in Tumor Progression: Signaling Functions of Cell Adhesion Molecules. 2009;1014(1):5866.

9. Liu GL, Yang HJ, Liu T, Lin YZJAPJoTM. Expression and significance of E-cadherin, N-cadherin, transforming growth factor- $\beta 1$ and Twist in prostate cancer. 2014;000(1):76-82.

10. Lu, Chen, Teresita, Mu?oz-Antonia, Douglas, One CJP. Trim28 contributes to EMT via regulation of E-cadherin and N-cadherin in lung cancer cell lines. 2014.

11. Ye Z, Ming Z, Tian B, Wu B, Li JJIJoC, Medicine E. Expression of IncRNA-CCAT1, E-cadherin and N-cadherin in colorectal cancer and its clinical significance. 2015;8(3):3707.

12. Lagendijk AK, Hogan BMJCTiDB. VE-cadherin in Vascular Development: A Coordinator of Cell Signaling and Tissue Morphogenesis. 2015;112:325-52.

13. Wallez Y, Vilgrain I, Huber PJTiCM. Angiogenesis: The VE-Cadherin Switch. 2006;16(2):55-9.

14. Fry SA, Robertson CE, Swann R, Dwek MV. Cadherin-5: a biomarker for metastatic breast cancer with optimum efficacy in oestrogen receptor-positive breast cancers with vascular invasion. British journal of cancer. 2016;114(9):1019-26. doi:10.1038/bjc.2016.66.

15. Higuchi K, Inokuchi M, Takagi Y, Ishikawa T, Otsuki S, Uetake H et al. Cadherin 5 expression correlates with poor survival in human gastric cancer. Journal of clinical pathology. 2017;70(3):217-21. doi:10.1136/jclinpath-2016-203640.

16. Mao XG, Xue XY, Wang L, Zhang X, Yan M, Tu YY et al. CDH5 is specifically activated in glioblastoma stemlike cells and contributes to vasculogenic mimicry induced by hypoxia. 2013;15(7):865-79.

17. Hess AR, Seftor EA, Gruman LM, Kinch MS, Seftor REB, Hendrix MJCJCB et al. VE-cadherin regulates EphA2 in aggressive melanoma cells through a novel signaling pathway: Implications for vasculogenic mimicry. 2006;5(2):228-33.

18. Zhao Y, Wang P, Meng J, Ji Y, Xu D, Chen T et al. MicroRNA-27a-3p Inhibits Melanogenesis in Mouse Skin Melanocytes by Targeting Wnt3a. 2015;16(5):10921-33.

19. Li W, Gao YJKJoMS. MiR-217 is involved in the carcinogenesis of gastric cancer by down-regulating CDH1 expression. 2018;34(7):37784.

20. Ma T, Zhao Y, Wei K, Yao G, Pan C, Liu B et al. MicroRNA-124 Functions as a Tumor Suppressor by Regulating CDH2 and EpithelialMesenchymal Transition in Non- Small Cell Lung Cancer. 2016;38(4):1563-74.

21. Zhou X, Huang H, Cui W, Wang Y, experimental XZJMemimjo, research c. Expression and Prognostic Significance of Cadherin 4 (CDH4) in Renal Cell Carcinoma. 2020;26:e922836.

22. Xiaoli Z, Yawei W, Lianna L, Haifeng L, Hui Z. Screening of Target Genes and Regulatory Function of miRNAs as Prognostic Indicators for Prostate Cancer. Medical science monitor : international medical journal of experimental and clinical research. 2015;21:3748-59. doi:10.12659/msm.894670. 
23. Zhang CJ, Du HJ. Screening key miRNAs for human hepatocellular carcinoma based on miRNA-mRNA functional synergistic network. Neoplasma. 2017;64(6):816-23. doi:10.4149/neo_2017_602.

24. Abraham S, Yeo M, Montero-Balaguer M, Paterson H, Dejana E, Marshall CJ et al. VE-Cadherin-mediated cell-cell interaction suppresses sprouting via signaling to MLC2 phosphorylation. Current biology : CB. 2009;19(8):668-74. doi:10.1016/j.cub.2009.02.057.

25. Peng H, Wang X, Zhang P, Sun T, Xia ZJIJoC, Pathology E. miR-27a promotes cell proliferation and metastasis in renal cell carcinoma. 2015;8(2):2259-66.

26. Berx G, Van Roy FJCSHPiB. Involvement of Members of the Cadherin Superfamily in Cancer. 2009;1(6).

27. Mao X, Xue X, Wang L, Zhang X, Yan M, Tu Y et al. CDH5 is specifically activated in glioblastoma stemlike cells and contributes to vasculogenic mimicry induced by hypoxia. 2013;15(7):865-79.

28. Yucel El, Sahin MJMBR. Fenretinide reduces angiogenesis by downregulating CDH5, FOXM1 and eNOS genes and suppressing microRNA-10b. 2020;47(3):1649-58.

29. Lufeng, Zheng, Zhiting, Zhang, Shufang, Qianqian et al. RNA Binding Protein RNPC1 Inhibits Breast Cancer Cell Metastasis via Activating STARD13-Correlated ceRNA Network. 2018.

30. Pan Y, Wei L, Wu X, Huo F, Mou J, Pei DJCD et al. MiR-106a-5p inhibits the cell migration and invasion of renal cell carcinoma through targeting PAK5. 2017;8(10).

31. Braga EA, Fridman MV, Loginov VI, Dmitriev AA, Morozov SGJFiG. Molecular Mechanisms in Clear Cell Renal Cell Carcinoma: Role of miRNAs and Hypermethylated miRNA Genes in Crucial Oncogenic Pathways and Processes. 2019;10.

32. Wang X, Tang S, Le SY, Lu R, Rader JS, Meyers C et al. Aberrant Expression of Oncogenic and Tumor-Suppressive MicroRNAs in Cervical Cancer Is Required for Cancer Cell Growth. 2008;3.

33. Tao L, Hua T, Lang Y, Min L, Xin LJCL. MicroRNA-27a functions as an oncogene in gastric adenocarcinoma by targeting prohibitin. 2009;273(2):0-242.

34. Guttilla, Irene, K., White, Bruce, Chemistry AJJoB. Coordinate Regulation of FOXO1 by miR-27a, miR-96, and miR-182 in Breast Cancer Cells. 2009.

35. Zhou L, Liang X, Zhang L, Yang L, Nagao N, Wu H et al. MiR-27a-3p functions as an oncogene in gastric cancer by targeting BTG2. 2016;7(32):51943-54.

36. Su C, Huang DP, Liu JW, Liu WY, Cao YOJOL. miR-27a-3p regulates proliferation and apoptosis of colon cancer cells by potentially targeting BTG1. 2019;18(3):2825-34.

37. Rao X, Wan L, Jie Z, Zhu X, Yin J, Cao HJO et al. Upregulated miR-27a-3p Indicates a Poor Prognosis in Pancreatic Carcinoma Patients and Promotes the Angiogenesis and Migration by Epigenetic Silencing of GATA6 and Activating VEGFA/VEGFR2 Signaling Pathway. 2019;12:11241-54.

38. Li L, Luo ZJOR. Dysregulated miR-27a-3p promotes nasopharyngeal carcinoma cell proliferation and migration by targeting Mapk10. 2017;37(5):2679-87.

39. Yan X, Yu H, Liu Y, Hou J, research YZJTic, treatment. miR-27a-3p Functions as a Tumor Suppressor and Regulates Non-Small Cell Lung Cancer Cell Proliferation via Targeting HOXB8. 2019;18(2):153303381986197.

40. Zhao N, Sun H, Sun B, Zhu D, Zhao X, Wang Y et al. miR-27a-3p suppresses tumor metastasis and VM by down-regulating VE-cadherin expression and inhibiting EMT: an essential role for Twist-1 in HCC. 2016;6:23091.

41. Giannotta M, Trani M, Dejana E. VE-cadherin and endothelial adherens junctions: active guardians of vascular integrity. Dev Cell. 2013;26(5):441-54. doi:10.1016/j.devcel.2013.08.020.

42. Labelle M, Schnittler HJ, Aust DE, Friedrich K, Breier GJCR. Vascular Endothelial Cadherin Promotes Breast Cancer Progression via Transforming Growth Factor Signaling. 2008;68(5):1388-97.

43. Hagendoorn J, Tong R, Fukumura D, Lin Q, Lobo J, Padera TP et al. Onset of Abnormal Blood and Lymphatic Vessel Function and Interstitial Hypertension in Early Stages of Carcinogenesis. 2006;66(7):3360-4.

44. Jiang J, Tang YL, Liang XH. EMT: a new vision of hypoxia promoting cancer progression. Cancer Biol Ther. 2011;11(8):714-23. doi:10.4161/cbt.11.8.15274.

45. Li X, Zheng L, Zhang F, Hu J, Chou J, Liu Y et al. STARD13-correlated ceRNA network inhibits EMT and metastasis of breast cancer. $2016 ; 7(17)$.

\section{Tables}

Table1. The basic features of the $\mathbf{3 0}$ microarrays of CDH5 expression profiling included in this study 


\begin{tabular}{|c|c|c|c|c|c|c|c|c|c|c|}
\hline \multirow[t]{2}{*}{ ID } & \multirow{2}{*}{ Author } & Publish & \multirow{2}{*}{ Country } & Sample & Cancer & Cancer & Cancer & Normal & Normal & Normal \\
\hline & & Year & & Type & $\mathrm{N}$ & M & SD & $\mathrm{N}$ & M & SD \\
\hline GSE40435 & 2012 & France & Magdalena,Wozniak & tissue & 101 & 10.1650 & 0.7566 & 101 & 8.6967 & 0.2687 \\
\hline GSE3 & 2001 & Germany & Wolfgang,Huber & tissue & 65 & 13.9696 & 2.6541 & 56 & 12.2923 & 2.7802 \\
\hline GSE53757 & 2014 & USA & John,A,Copland & tissue & 72 & 10.0568 & 0.7638 & 72 & 9.0084 & 0.4828 \\
\hline GSE46699 & 2013 & USA & Daniel,J,Serie & tissue & 67 & 7.9352 & 1.2416 & 63 & 6.2838 & 0.6158 \\
\hline GSE15641 & 2009 & USA & Jon,Jones & tissue & 32 & 7.9016 & 0.7804 & 23 & 7.7931 & 0.1774 \\
\hline GSE105261 & 2017 & USA & Sunil,Sudarshan & tissue & 35 & 9.2945 & 0.7933 & 9 & 8.7874 & 0.6606 \\
\hline GSE40911 & 2012 & USA & Angela,A,Fachel & tissue & 22 & 3.7543 & 1.0225 & 22 & 2.5789 & 0.9541 \\
\hline GSE40911 & 2012 & USA & Angela,A,Fachel & tissue & 22 & 3.7543 & 1.0225 & 22 & 2.5789 & 0.9541 \\
\hline GSE36895 & 2012 & USA & Samuel,Pena-Llopis & tissue & 23 & 8.7739 & 0.7227 & 23 & 7.5138 & 0.4702 \\
\hline GSE16449 & 2009 & USA & $\mathrm{A}, \mathrm{R}, \mathrm{Brannnon}$ & tissue & 52 & 1.3813 & 1.3813 & 18 & 1.7967 & 0.8965 \\
\hline GSE16441 & 2009 & USA & Huiqing,Liu & tissue & 17 & 2.2440 & 0.7618 & 17 & 1.8928 & 0.8231 \\
\hline GSE53000 & 2014 & USA & Pierre,Martinez & tissue & 56 & 10.3342 & 0.6073 & 6 & 9.4014 & 0.2738 \\
\hline GSE66272 & 2015 & USA & Klaus,Jung & tissue & 26 & 0.8218 & 0.9534 & 26 & -0.2236 & 0.5518 \\
\hline GSE68417 & 2015 & USA & Bryan,J,Thibodeau & tissue & 29 & 8.2498 & 1.1903 & 14 & 7.2510 & 0.6557 \\
\hline GSE6344 & 2006 & USA & John,A,Copland & tissue & 10 & 9.7863 & 0.3465 & 10 & 7.9947 & 0.9520 \\
\hline GSE781 & 2003 & USA & Affymetrix, Inc & tissue & 12 & 9.1342 & 1.1568 & 5 & 7.9188 & 0.8022 \\
\hline GSE76351 & 2015 & Russia & Sergey,Solodskikh & tissue & 12 & 8.7220 & 0.9786 & 12 & 6.5342 & 0.1994 \\
\hline GSE14762 & 2009 & USA & Karl,Dykema & tissue & 10 & 9.4894 & 0.9681 & 12 & 9.2252 & 0.4275 \\
\hline GSE17895 & 2009 & USA & Karl,J,Dykema & tissue & 151 & 8.4973 & 1.3091 & 9 & 7.2854 & 0.7367 \\
\hline GSE17818 & 2009 & USA & Karl,Dykema & tissue & 102 & 8.7651 & 1.2894 & 13 & 8.0411 & 0.4616 \\
\hline GSE17816 & 2009 & USA & Karl,Dykema & tissue & 36 & 7.9034 & 1.3510 & 9 & 7.2854 & 0.7367 \\
\hline GSE71963 & 2015 & JAPAN & Mika,Takahashi & tissue & 32 & 2.6268 & 1.5455 & 16 & 1.0341 & 0.4820 \\
\hline GSE72922 & 2015 & Italy & Fabio,Sallustio & tissue & 12 & 6.5574 & 0.4431 & 11 & 6.6631 & 0.6927 \\
\hline GSE100666 & 2017 & China & Zhiqiang,Peng & tissue & 3 & 12.7572 & 0.2469 & 3 & 11.1296 & 0.1608 \\
\hline GSE47032 & 2013 & Italy & Alessio,Valletti & tissue & 10 & 7.2094 & 1.1292 & 10 & 5.7553 & 0.3927 \\
\hline GSE14994 & 2009 & USA & Rameen,Beroukhim & tissue & 90 & 9.3803 & 0.6720 & 48 & 9.2423 & 0.4061 \\
\hline GSE26574 & 2011 & USA & Karl,Dykema & tissue & 8 & 11.0338 & 0.4724 & 8 & 9.5086 & 0.5717 \\
\hline GSE11151 & 2008 & Netherlands & Dmitry,Zubakov & tissue & 26 & 10.4357 & 0.8631 & 5 & 10.1712 & 0.5222 \\
\hline GSE66270 & 2015 & Germany & Klaus,Jung & tissue & 14 & 1.1472 & 0.6860 & 14 & -0.2821 & 0.2033 \\
\hline GSE66271 & 2016 & Germany & Klaus,Jung & tissue & 12 & 0.4421 & 1.1035 & 12 & -0.1611 & 0.7955 \\
\hline GSE77199 & 2016 & $\begin{array}{l}\text { United } \\
\text { Kingdom }\end{array}$ & Joseph,Wragg & tissue & 4 & 9.2530 & 0.6968 & 4 & 9.0196 & 0.9683 \\
\hline
\end{tabular}

Table2. Relationship between the expression of CDH5 and clinicopathological parameters in ccRCC. 


\begin{tabular}{|c|c|c|c|c|c|}
\hline \multicolumn{2}{|c|}{ Clinicopathological parameters } & \multicolumn{4}{|c|}{ Relevant expression of CDH5 } \\
\hline & & $\mathrm{n}$ & Mean $\pm S D$ & $\mathrm{t}$ & $p$ value \\
\hline \multirow[t]{2}{*}{ Tissue } & normal & 72 & $10.5024 \pm 0.51025$ & 14.482 & $<0.001 * \star \star$ \\
\hline & KIRC & 533 & $11.5959 \pm 1.05656$ & & \\
\hline \multirow[t]{2}{*}{ Age } & $₫ 60$ & 246 & $11.6253 \pm 1.09235$ & 0.591 & 0.591 \\
\hline & $\geq 60$ & 288 & $11.5710 \pm 1.02437$ & & \\
\hline \multirow[t]{2}{*}{ Gender } & male & 346 & $11.5980 \pm 1.01013$ & 0.565 & 0.953 \\
\hline & female & 188 & $11.5924 \pm 1.13726$ & & \\
\hline \multirow[t]{2}{*}{$\mathrm{T}$} & T1-T2 & 343 & $11.6747 \pm 1.06626$ & 2.404 & $0.016 *$ \\
\hline & T3-T4 & 191 & $11.4546 \pm 1.02373$ & & \\
\hline \multirow[t]{2}{*}{$\mathrm{N}$} & NO & 240 & $11.5242 \pm 1.08271$ & 2.670 & $0.008^{\star *}$ \\
\hline & YES & 16 & $10.7736 \pm 1.17482$ & & \\
\hline \multirow[t]{2}{*}{ M } & NO & 422 & $11.6575 \pm 1.00230$ & 3.212 & 0.001 ** \\
\hline & YES & 79 & $11.2522 \pm 1.16205$ & & \\
\hline \multirow[t]{2}{*}{ Pathologic stage } & $\mathrm{I}-\mathrm{II}$ & 325 & $11.6984 \pm 1.05057$ & 2.807 & $0.005^{\star \star}$ \\
\hline & III-IV & 207 & $11.4363 \pm 11.43632$ & & \\
\hline
\end{tabular}

SD: standard deviation; ccRCC: clear cell renal carcinoma

*p<0.05 was considered statistically significant.

Table3. Relationship between the expression of CDH5 and clinicopathological parameters in ccRCC.

\begin{tabular}{|c|c|c|c|c|c|}
\hline \multicolumn{6}{|c|}{ Characteristic } \\
\hline & & $\mathrm{n}$ & Mean $\pm S D$ & $\mathrm{t}$ & $\mathrm{p}$ value \\
\hline \multirow[t]{2}{*}{ Tissue } & normal & 17 & $9.2647 \pm 2.25074$ & 16.576 & $\triangle 0.001 * \star \star$ \\
\hline & KIRC & 17 & $0.0882 \pm 0.36380$ & & \\
\hline \multirow[t]{2}{*}{ Age } & $\nabla 60$ & 9 & $9.5833 \pm 2.40780$ & 0.606 & 0.553 \\
\hline & $\geq 60$ & 8 & $8.9062 \pm 2.16241$ & & \\
\hline \multirow[t]{2}{*}{ Gender } & male & 12 & $9.3041 \pm 2.19653$ & 0.108 & 0.915 \\
\hline & female & 5 & $9.1700 \pm 2.64187$ & & \\
\hline \multirow{2}{*}{$\begin{array}{l}\text { Tumor } \\
\text { diameter }\end{array}$} & $\leq 5$ & 10 & $9.0000 \pm 2.55419$ & -0.567 & 0.579 \\
\hline & $\bigotimes 5$ & 2 & $9.6428 \pm 1.85459$ & & \\
\hline \multirow{2}{*}{$\begin{array}{l}\text { Clinical } \\
\text { Stage }\end{array}$} & $\mathrm{I}-\mathrm{II}$ & 9 & $9.0833 \pm 2.71546$ & -0.343 & 0.737 \\
\hline & III-IV & 8 & $9.4687 \pm 1.74988$ & & \\
\hline \multirow[t]{2}{*}{ T stage } & T1 & 15 & $9.1900 \pm 2.60955$ & -0.346 & 0.721 \\
\hline & T2-T3 & 2 & $3.5509 \pm 3.09799$ & & \\
\hline
\end{tabular}


Table4. Relationship between the expression of CDH5 miRNA and clinicopathological parameters in ccRCC.

\begin{tabular}{|c|c|c|c|c|c|}
\hline \multicolumn{2}{|c|}{ Clinicopathological parameters } & \multicolumn{4}{|c|}{ Relevant expression of CDH5 miRNA } \\
\hline & & $\mathrm{n}$ & Mean $\pm S D$ & $\mathrm{t}$ & $\mathrm{p}$ value \\
\hline \multirow[t]{2}{*}{ Tissue } & normal & 70 & $8.9564 \pm 0.6323$ & \multirow[t]{2}{*}{7.674} & \multirow[t]{2}{*}{$<0.001^{\star \star \star}$} \\
\hline & KIRC & 240 & $9.5362 \pm 0.5348$ & & \\
\hline \multirow[t]{2}{*}{ Age } & $₫ 60$ & 118 & $9.5085 \pm 0.5417$ & \multirow[t]{2}{*}{-0.789} & \multirow[t]{2}{*}{0.431} \\
\hline & $\geq 60$ & 122 & $9.5630 \pm 0.5290$ & & \\
\hline \multirow[t]{2}{*}{ Gender } & male & 159 & $9.5983 \pm 0.5176$ & \multirow[t]{2}{*}{2.548} & \multirow[t]{2}{*}{$0.011^{*}$} \\
\hline & female & 81 & $9.4143 \pm 0.5504$ & & \\
\hline \multirow[t]{2}{*}{$\mathrm{T}$} & $\mathrm{T} 1-\mathrm{T} 2$ & 153 & $9.4783 \pm 0.5497$ & \multirow[t]{2}{*}{-2.244} & \multirow[t]{2}{*}{$0.026^{*}$} \\
\hline & T3-T4 & 87 & $9.6381 \pm 0.4944$ & & \\
\hline \multirow[t]{2}{*}{$N$} & NO & 97 & $9.4639 \pm 0.5576$ & \multirow[t]{2}{*}{-1.723} & \multirow[t]{2}{*}{0.085} \\
\hline & YES & 143 & $9.5853 \pm 0.5150$ & & \\
\hline \multirow[t]{2}{*}{ M } & NO & 169 & $9.4561 \pm 0.5392$ & \multirow[t]{2}{*}{0.491} & \multirow[t]{2}{*}{$<0.001^{\star \star \star}$} \\
\hline & YES & 69 & $9.7188 \pm 0.4770$ & & \\
\hline \multirow[t]{2}{*}{ Pathologic stage } & $\mathrm{H}-\mathrm{II}$ & 145 & $9.4595 \pm 0.5409$ & \multirow[t]{2}{*}{0.745} & \multirow[t]{2}{*}{$<0.007 * \star$} \\
\hline & III-IV & 94 & $9.6485 \pm 0.5068$ & & \\
\hline
\end{tabular}

SD: standard deviation; ccRCC: clear cell renal carcinoma

${ }^{*} \mathrm{p}<0.05$ was considered statistically significant.

\section{Figures}


a

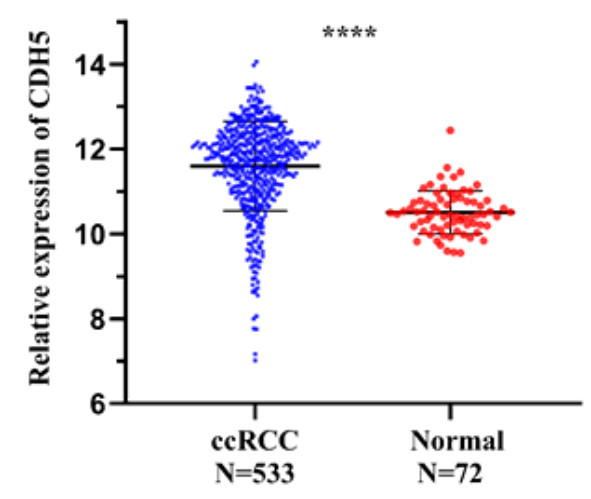

b

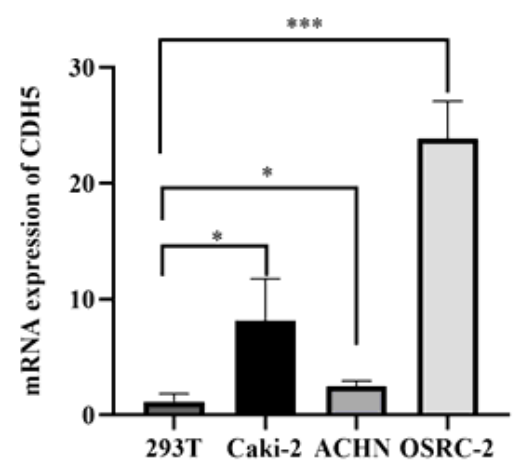

c

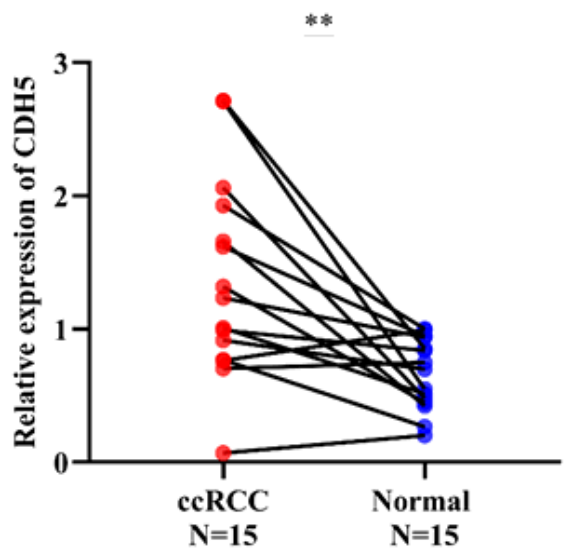

Figure 1

Upregulation of CDH5 mRNA in clear cell renal cell carcinoma (ccRCC). (a) The mRNA expression of CDH5 was significantly evaluated in

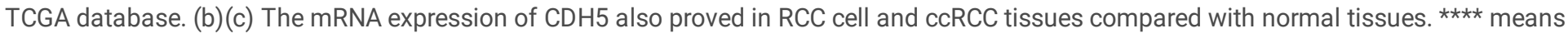
$\mathrm{p}<0.0001$ 

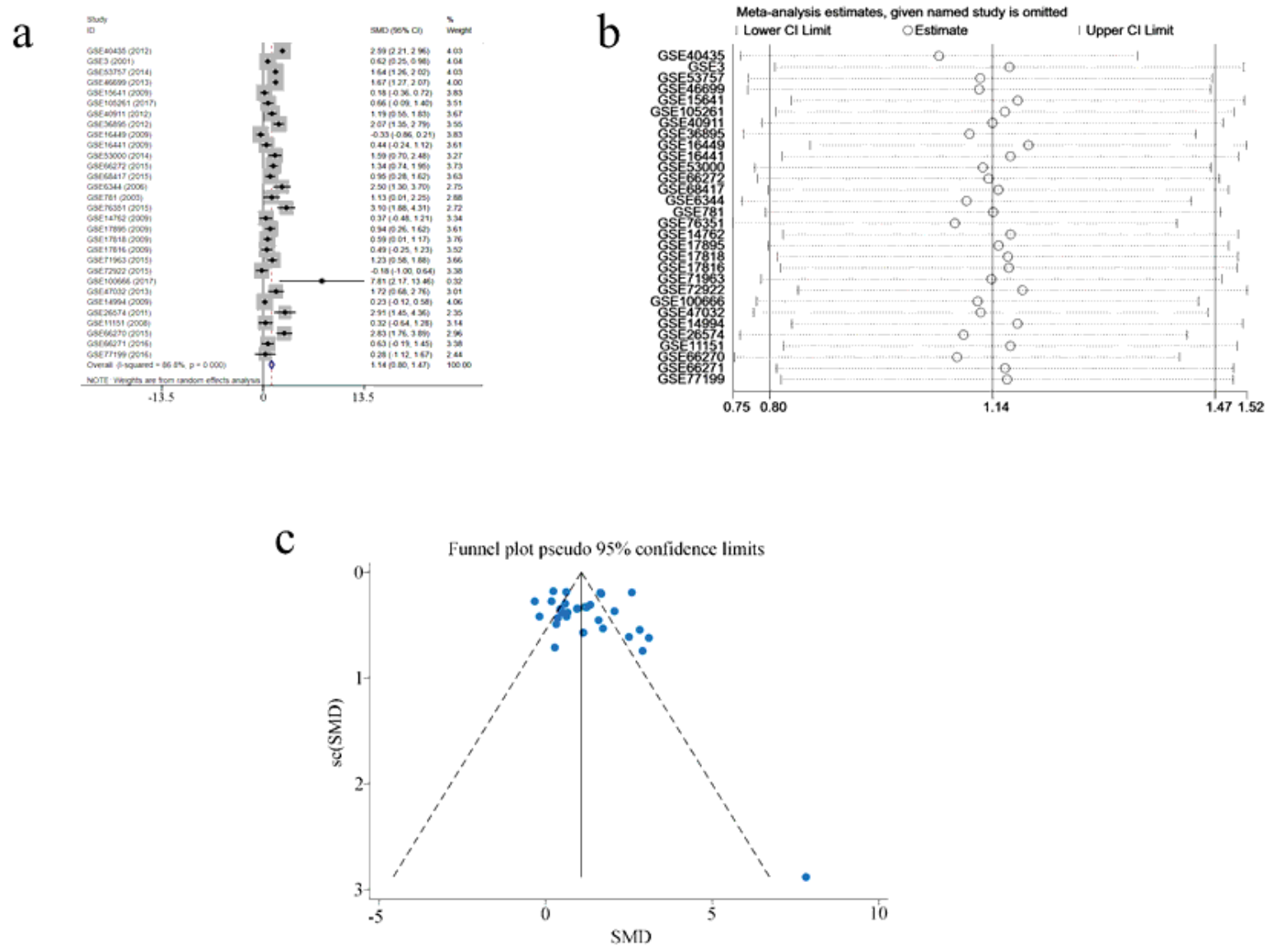

\section{Figure 2}

The Meta-analysis of CDH5 mRNA expression in ccRCC. (a) The total of SMDs with 95\% Cl accounted for 1.07 (0.96, 1.19). ccRCC tissue subgroup was highly heterogeneous $(\mathrm{I} 2=86.8 \%$, $\mathrm{p}<0.001)$. (b). A meta-analysis of CDH5 expression in ccRCC showed no significant difference in the sensitivity analysis. (c). A meta-analysis of CDH5 expression in ccRCC using Begg funnel map. Symmetric Begg funnel map indicated no publication bias $(p=0.116)$. 


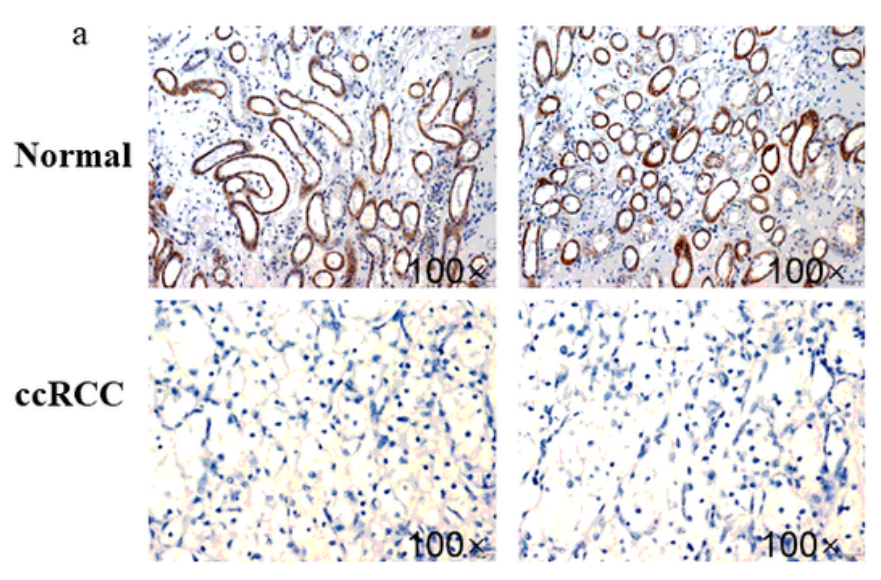

b

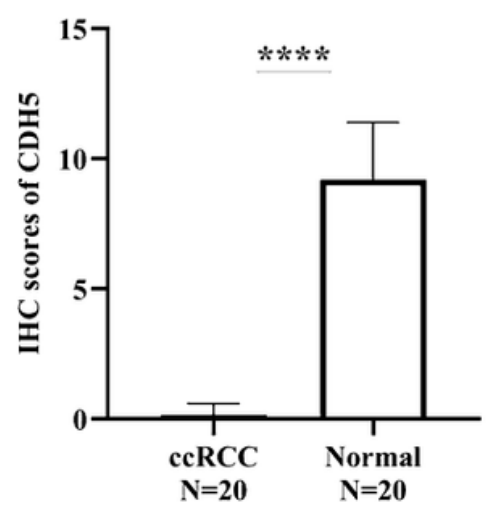

Figure 3

The protein of $\mathrm{CDH} 5$ was mainly distributed on the proximal convoluted tubule of adjacent normal kidney samples. (a) Representative slides of ccRCC tissues and matched control tissues, stained by an anti-CDH5 antibody. (b) The expression level of CDH5 protein in ccRCC and

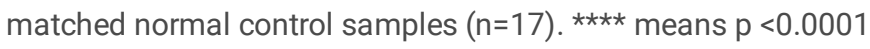



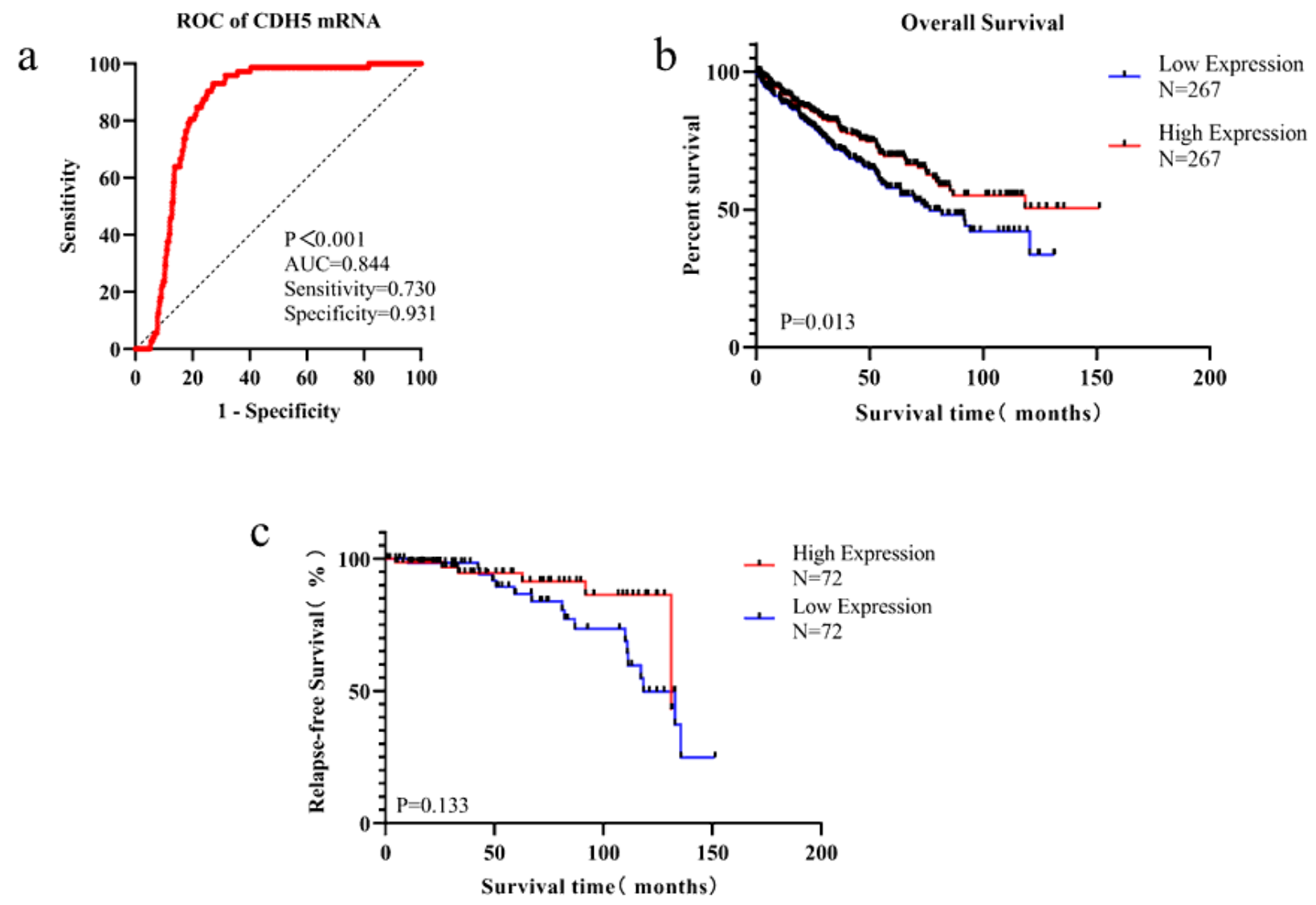

\section{Figure 4}

The ROC curve was used to assess the diagnostic efficacy of CDH5 mRNA, the prognostic value of CDH5 mRNA was evaluated by OS and DFS. (a). The ROC curve for evaluating the diagnostic performance of CDH5 in 533 cases of ccRCC and 72 cases of normal kidney tissues based on the TCGA database. The AUC was 0.844. (b)(c). Potential prognostic value of CDH5 mRNA expression in ccRCC patients. 
a
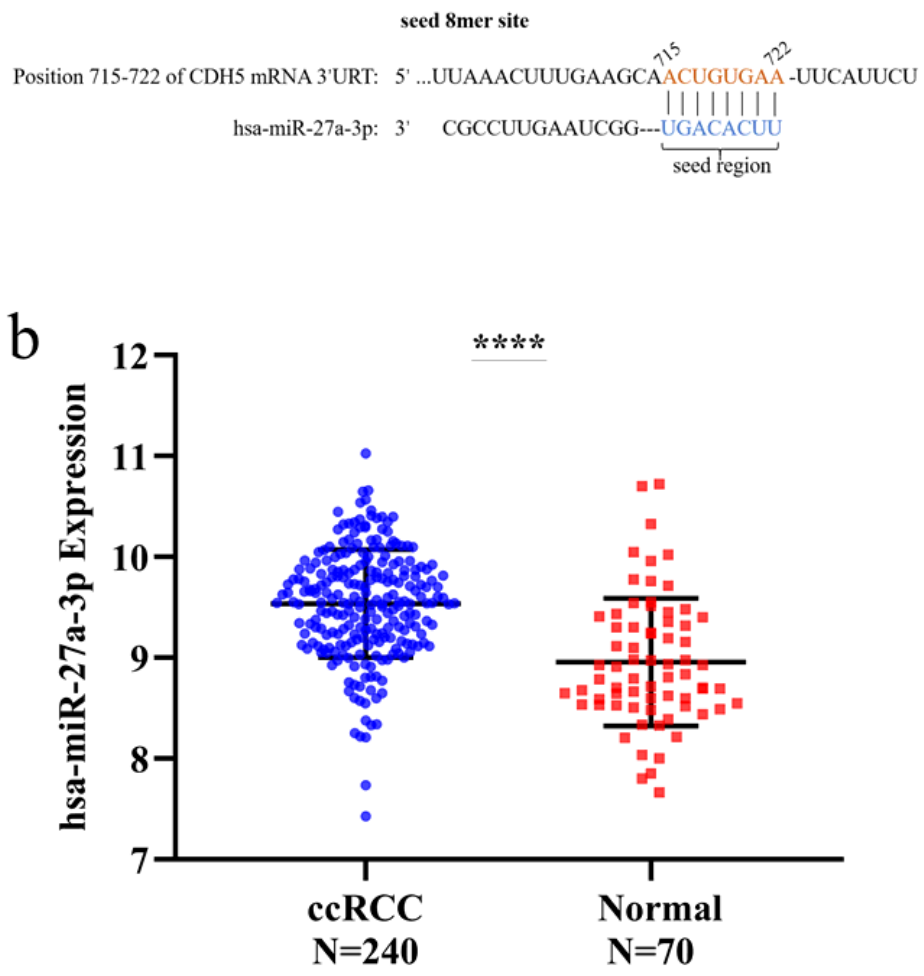

\section{Figure 5}

Mir-27a-3p targeting CDH5 at the post-transcriptional level. (a). Mir-27a-3p as a highly conserved seed region miRNA of CDH5. (b). The miRNA

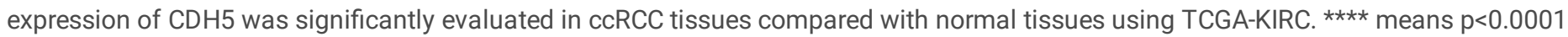



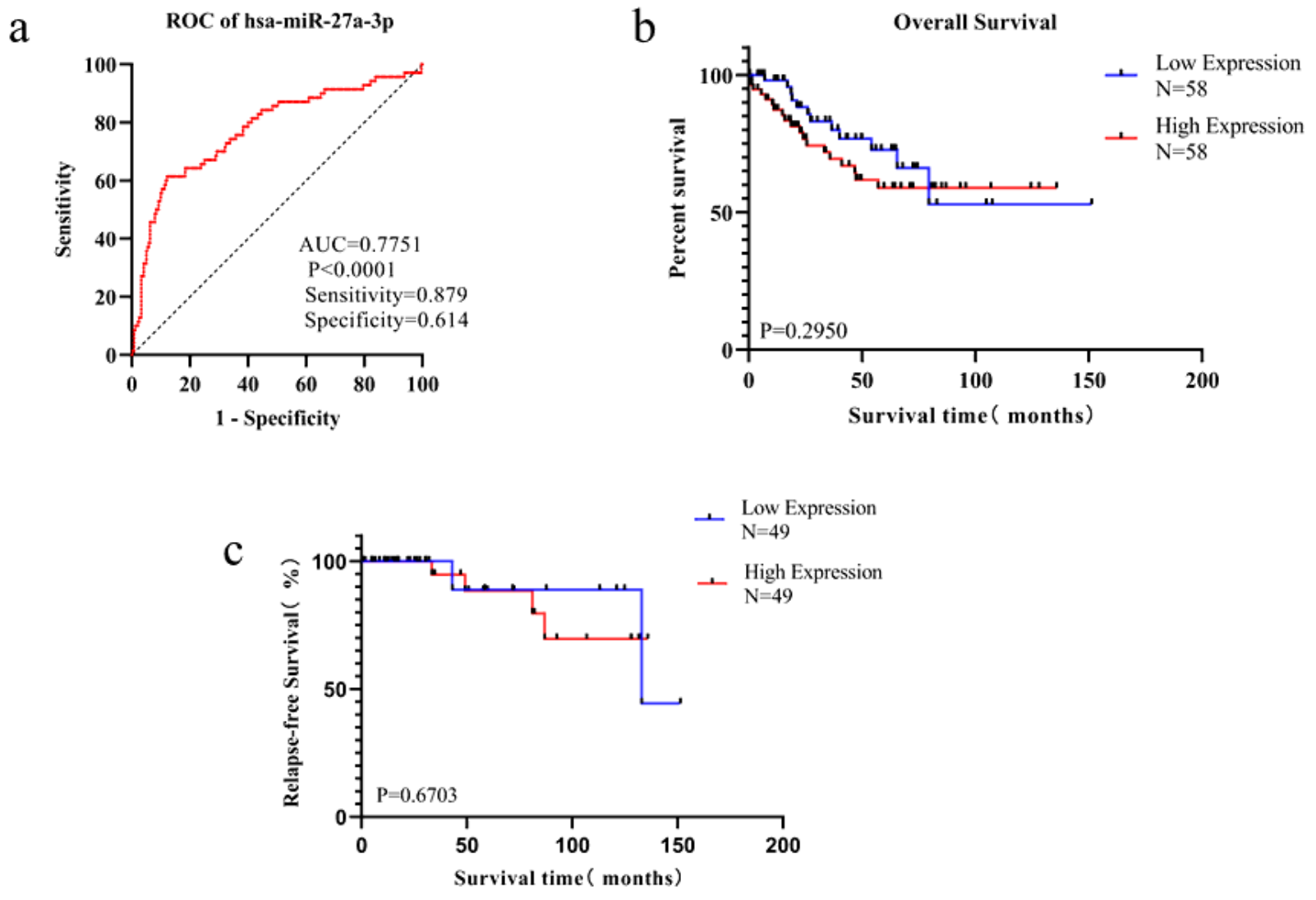

\section{Figure 6}

The ROC curve was used to assess the diagnostic efficacy of has-27a-3p, prognostic value was evaluated by OS and DFS. (a) The ROC curve for evaluating the diagnostic performance of miRNA in 240 cases of CCRCC and 70 cases of normal kidney tissues based on the TCGA database. The AUC was 0.775. (b)(c). Potential prognostic value of CDH5 miRNA expression in ccRCC patients in the TCGA database. 